\title{
Exigências nutricionais e crescimento de plantas de mogno (Swietenia macrophylla King.)
}

\author{
Cacilda Adélia Sampaio de SOUZA ${ }^{1}$, Carlos Alberto Franco TUCCI², José Ferreira da SILVA², Weslei Ortiz \\ RIBEIRO $^{4}$
}

\section{RESUMO}

A necessidade de desenvolver pesquisas referentes aos requerimentos nutricionais das espécies florestais, principalmente as essências florestais nativas da Amazônia, como o mogno, é inadiável, visto que as constantes exploraçóes de espécies de alto valor econômico são cada vez maiores. Com o objetivo de avaliar o crescimento e o requerimento nutricional de mudas de mogno, foi realizado um experimento em casa de vegetaçáo com 14 tratamentos. O delineamento experimental foi de blocos casualisados, com 5 repetições. As características de crescimento avaliadas foram: altura da parte aérea, diâmetro de colo, matéria seca da parte aérea, da raiz e total, relação raiz e parte aérea e conteúdo de nutrientes da matéria seca da parte aérea. Os resultados obtidos permitem concluir que: é necessária a correção conjunta da acidez e da fertilidade do solo para solos ácidos e de baixa fertilidade natural, mesmo que o teor de matéria orgânica seja considerado alto; dos nutrientes, P é o que mais limita o desenvolvimento da planta, bem como a absorção dos outros nutrientes; não há necessidade de adição de $\mathrm{K}$ para o desenvolvimento das plantas quando se realiza calagem; na ausência de calagem a adição de K deve ser recomendada e o requerimento nutricional do mogno em macronutrientes obedece à ordem decrescente de: $\mathrm{P}>\mathrm{S}>\mathrm{K}>\mathrm{N}$.

PALAVRAS-CHAVE: Nutrição florestal, produção de mudas e fertilizantes.

\section{Nutritional requirements and growth of mahogany (Swietenia macrophylla King.) plants}

\begin{abstract}
The need for developing research related principally to the nutritional requirements of essential native Amazonian forest species, such as mahogany, is urgent, since the exploration of highly valuable species is continually more frequent. With the objective of evaluating the growth and the nutritional requirement of mahogany plants, an experiment in a vegetation house with 14 treatments was undertaken. The experiment outline was of occasional blocks with 5 repetitions. The growth characteristics evaluated were: height of the aerial part, diameter of the bottom, dry material of the aerial part, root and total, relationship of root and aerial part, relationship and nutrient content of dry material of the aerial part. The results lead to the conclusion that: the conjoint correction of acidity and the fertility of the soil for acid soils and low natural fertility is necessary even if the content of the organic material is considered high; of the nutrients, $\mathrm{P}$ is the one which limits most the development of the plant, as well as the absorption of other nutrients; there is no need to add $\mathrm{K}$ for developing the plants when liming occurs.; addition of $\mathrm{K}$ is recommended when liming does not occur; and the nutritional requirement of mahogany in macronutrients obeys the decreasing order of $\mathrm{P}>\mathrm{S}>\mathrm{K} . \mathrm{N}$.
\end{abstract}

KEYWORDS: Forest nutritional, plants production and fertilizantion.

\footnotetext{
${ }^{1}$ Universidade Federal do Amazonas - UFAM. E-mail: adelia_sampaio@yahoo.com.br

2 Universidade Federal do Amazonas - UFAM. E-mail: ctucci@ufam.edu.br

${ }^{3}$ Universidade Federal do Amazonas - UFAM. E-mail: jferreira@ufam.edu.br

${ }^{4}$ Universidade Federal do Amazonas - UFAM. E-mail: weslei_ortiz@yahoo.com.br
} 


\section{INTRODUÇÃO}

Dentre as inúmeras espécies florestais nativas do Brasil exploradas em grande escala, se encontra o mogno (Swietenia macrophylla King.). O mogno é bastante explorado devido ao seu grande valor comercial em todo o mundo, seja pela beleza da madeira que produz, seja por suas características tecnológicas bastante apreciadas.

O conhecimento das exigências nutricionais em espécies florestais é de grande importância, pois é imprescindível no crescimento e na distribuição de biomassa, principalmente nos ecossistemas florestais da Amazônia, dada sua escassez na maioria dos solos desta regiáo; é um dos principais fatores que normalmente assume a maior importância na produção e que mais limita o aumento de produtividade das plantas (Sena, 2008; Silva et al, 2007, Tucci, 1991).

Segundo Sarcinelli et al. (2004) o conhecimento das exigências nutricionais em espécies florestais permite a identificaçáo e correçấo de deficiências que podem ocorrer em exemplares plantados em diferentes substratos degradados, possibilitando, dessa maneira, intervençóes corretas, sem desperdícios e de menor impacto ambiental. Assim, a correçâo das limitaçôes nutricionais do solo durante a formaçâo das mudas no campo é de grande importância ecológica e econômica em programas florestais.

A necessidade de desenvolver pesquisas referentes aos requerimentos nutricionais das espécies florestais, principalmente as essências florestais nativas da Amazônia, como o mogno, é inadiável, uma vez que estas informaçóes são imprescindíveis para fornecer subsídios que garantam o manejo adequado da floresta.

Dessa forma, esta pesquisa teve como objetivo avaliar o crescimento e o requerimento nutricional de mudas de mogno em Latossolo Amarelo distrófico, na presença e ausência de calagem.

\section{MATERIAL E MÉTODOS}

O estudo foi realizado no período de abril/2006 a abril/2007 na Universidade Federal do Amazonas (UFAM). O solo foi coletado na camada 0 a $30 \mathrm{~cm}$ de profundidade e classificado como Latossolo Amarelo distrófico, de textura argilosa, cuja localização tem coordenadas UTM 21M016894E, $9657166 \mathrm{~N}$, no campus da referida Universidade. Em seguida foi destorroado, seco ao ar livre e, passado em peneira de malha de $4 \mathrm{~mm}$ de abertura. As análises químicas e textura foram feitas segundo EMBRAPA (1999) e estão expressos na Tabela 1 .

Foram avaliados os seguintes tratamentos: $\mathrm{T}_{1}-($ TEST) - Solo natural, Testemunha; $\mathrm{T}_{2}$ - (Mi) - Solo adubado com micronutrientes; $\mathrm{T}_{3}-$ (calMi) - Solo adubado com calagem e micronutrientes; $\mathrm{T}_{4}-$ (cal) - Solo adubado com calagem; $\mathrm{T}_{5}-(\mathrm{COM})-$ Solo adubado com N, P, K e S, calagem e micronutrientes; $\mathrm{T}_{6}-(\mathrm{COM}-\mathrm{N})$ - Solo adubado com P, K e $S$, calagem e micronutrientes; $\mathrm{T}_{7}$ - (COM-P) - Solo adubado com N, K e S, calagem e micronutrientes; $\mathrm{T}_{8}-(\mathrm{COM}-\mathrm{K})$ - Solo adubado com N, P, S, calagem e micronutrientes; $\mathrm{T}_{9}-(\mathrm{COM}-\mathrm{S})$ - Solo adubado com N, P e K, calagem e micronutrientes; $\mathrm{T}_{10}-(\mathrm{COM}$-cal) - Solo adubado com N, P, Ke $S$ e micronutrientes; $\mathrm{T}_{11}-(\mathrm{COM}$-cal-N) - Solo adubado com P, K e S e micronutrientes; $\mathrm{T}_{12}-(\mathrm{COM}$-cal-P) - Solo adubado com N, Ke S e micronutrientes; $\mathrm{T}_{13}-(\mathrm{COM}$-cal-K) - Solo adubado com N, P e S e micronutrientes; e $\mathrm{T}_{14}$ (COM-cal-S) - Solo adubado com N, P e K e micronutrientes.

O delineamento experimental foi em blocos casualisados com 5 repetiçóes.

Nos tratamentos em que a calagem estava presente foi seguida a recomendação proposta por Silva (2004), isto é, $250 \mathrm{~g}$ de calcário por ton ${ }^{-1}$ de substrato e foi realizada com trinta dias de antecedência da aplicação dos demais tratamentos.

As doses aplicadas foram de 150, 450, 150 e $50 \mathrm{mg} \mathrm{kg}^{-1}$ de N, $\mathrm{P}_{2} \mathrm{O}_{5}, \mathrm{~K}_{2} \mathrm{O}$ e $\mathrm{S}$, respectivamente, adaptadas de Duboc et al. (1996). As fontes foram uréia, acido fosfórico, cloreto de potássio e sulfato de sódio, respectivamente, aplicados na forma de solução. A fonte de micronutrientes foi a FTE-Br 12 aplicada em dose equivalente a $50 \mathrm{~kg} 2000$ ton $^{-1}$ de solo ou $0,15 \mathrm{~g}$ por parcela experimental, conforme Silva Júnior (2006). Isto correspondeu à aplicação de 0,$45 ; 0,2 ; 0,75 ; 0,75 ; 0,025$ e 2,25 mg kg ${ }^{-1} \mathrm{de} \mathrm{B}, \mathrm{Cu}, \mathrm{Fe}, \mathrm{Mn}, \mathrm{Mo}$ e Zn, respectivamente.

As sementes de mogno foram coletadas de árvores situadas à Vila Militar (Colônia Oliveira Machado) em Manaus, AM. Os frutos foram coletados de árvores matrizes selecionadas de acordo com seu estado fitossanitário. Para a produçấo de mudas as sementes foram colocadas para germinar em bandejas com tubetes, utilizando-se como substrato areia. Ao atingirem $12 \mathrm{~cm}$ de altura (2 pares de folhas), as plântulas foram selecionadas e transplantadas para os vasos plásticos com capacidade de $6 \mathrm{~kg}$.

Tabela 1 - Características químicas e teor de argila do solo, antes da incorporação do calcário e aplicação dos tratamentos.

\begin{tabular}{|c|c|c|c|c|c|c|c|c|c|c|c|c|c|c|c|c|}
\hline $\begin{array}{l}\mathrm{Ph} \\
\mathrm{H}_{2} \mathrm{O}\end{array}$ & $\mathrm{H}+\mathrm{Al}$ & $\mathrm{Al}^{3+}$ & $\mathrm{Ca}^{2+}$ & $\mathrm{Mg}^{2+}$ & $\mathrm{T}$ & $t$ & $P$ & K & $\mathrm{Fe}$ & $\mathrm{Cu}$ & $\mathrm{Mn}$ & $\mathrm{Zn}$ & $\mathrm{m}$ & V & Argila & M.O \\
\hline & -----. & -------- & $---\mathrm{cmo}$ & $\mathrm{Kg}^{-1}-\cdots$ & ------ & ------ & & --- & --- & $\mathrm{Mg} \mathrm{Kg}$ & ------. & ----- & --------- & ---\%-- & ------ & $-\mathrm{g} \mathrm{kg}^{-1}$ \\
\hline 4,34 & 8,41 & 0,98 & 0,91 & 0,15 & 9,55 & 2,12 & 5 & 30 & 53 & 0,14 & 5,47 & 1,77 & 46,22 & 11,94 & 83,76 & 43,44 \\
\hline
\end{tabular}


A primeira adubaçáo nitrogenada foi realizada 10 dias após o transplantio com $50 \%$ da dose total de N. A segunda foi aplicada 30 dias depois.

As plântulas de mogno permaneceram em casa de vegetação por um período de 120 dias. Foram avaliadas a altura da parte aérea, diâmetro de colo, matéria seca da parte aérea (MSPA), da raiz (MSR) e total (MST), relação raiz e parte aérea $(\mathrm{R} / \mathrm{PA})$, o teor de nutrientes da matéria seca da parte aérea. $\mathrm{O}$ teor de nutrientes da matéria seca da parte aérea seguiu metodologia proposta por Malavolta et al. (1997).

\section{RESULTADOS E DISCUSSÃO}

\section{CRESCIMENTO DAS MUDAS}

$\mathrm{O}$ crescimento em altura e diâmetro, e a produção de matéria seca da parte aérea, raiz e total das mudas de mogno foram limitados quando náo se realizou a correçáo conjunta da acidez e da fertilidade do solo com macro e micronutrientes, pois os tratamentos em que o substrato náo foi corrigido $\left(\mathrm{T}_{1}\right.$, $\mathrm{T}_{2}, \mathrm{~T}_{3}$ e $\left.\mathrm{T}_{4}\right)$ apresentaram valores inferiores ao completo $\left(\mathrm{T}_{5}\right)$ (Tabela 2).

Em relação ao completo $\left(\mathrm{T}_{5}\right)$, a omissão de $\mathrm{N}$ afetou de forma distinta as características de crescimento, com comportamento semelhante tanto na presença quanto na ausência da calagem ( $\mathrm{T}_{6} \mathrm{e} \mathrm{T}_{11}$, respectivamente). Não afetando a altura, a matéria seca da parte aérea e total, porém, favoreceu o diâmetro, a matéria seca de raiz e a relaçấo R/PA (Tabela 2).

Os resultados que indicam a omissáo de $\mathrm{N}$ (na presença ou ausência da calagem), como fator não limitante ao crescimento das mudas de mogno, podem ser explicados através do teor de matéria orgânica encontrado no solo utilizado para compor substrato.

A Tabela 1 apresenta os resultados da análise de solo, e por meio desta foi observado que o teor de matéria orgânica encontrado foi de $43,44 \mathrm{~g} \mathrm{~kg}^{-1}$, sendo considerado de nível alto de acordo com a CFSEMG (1999). Este fato pode explicar a omissão do nutriente $\mathrm{N}$ não ter afetado o crescimento em altura e diâmetro e a produção de matéria seca da parte aérea, raiz e total das mudas de mogno. A matéria orgânica através do processo de mineralizaçáo pode ter fornecido uma quantidade suficiente de $\mathrm{N}$ para atender a demanda da espécie durante a fase experimental.

Segundo Sá (2001) o aumento da matéria orgânica do solo proporciona maior mineralizaçáo e disponibilidade de nitrogênio ao longo do tempo. De acordo Wietholter (2000) o aumento do teor de matéria orgânica do solo aumenta o teor de $\mathrm{N}$ no solo, uma vez que o teor de $\mathrm{N}$ da matéria orgânica é relativamente constante (de 5\%). Santos e Tom (2003) trabalhando com a disponibilidade de nutrientes no solo e sistema de manejo do solo verificaram que houve diferenças no teor de matéria orgânica entre as profundidades do solo, decrescendo progressivamente da camada 0-50 cm (29-38 g $\left.\mathrm{kg}^{-1}\right)$ para $15-20 \mathrm{~cm}\left(27-28 \mathrm{~g} \mathrm{~kg}^{-1}\right)$. Tendência semelhante foi observada por Sá (1993) com redução de $53 \mathrm{~g} \mathrm{~kg}^{-1}$ para $35 \mathrm{~g} \mathrm{~kg}^{-1}$ de matéria orgânica e por Santos et al. (1995) com redução variando de $27-33 \mathrm{~g} \mathrm{~kg}^{-1}$ para $23-25 \mathrm{~g} \mathrm{~kg}^{-1}$, nas mesmas camadas.

Um alto teor de matéria orgânica no substrato também pode ter interferido nos resultados obtidos por Oliveira et al. (1997) para o angelim-pedra (Dinizia excelsa Ducke).

Tabela 2 - Crescimento em altura, diâmetro, matéria seca da parte aérea, matéria seca da raiz, matéria seca total e relação raiz/parte aérea das plantas de mogno (Swietenia macrophylla King.) aos 120 dias após transplantio, submetidas a diferentes condições nutricionais.

\begin{tabular}{lcccccc}
\hline Tratamentos & Altura $(\mathrm{cm})$ & Diâmetro $(\mathrm{mm})$ & MSPA $(\mathrm{g})$ & MSR $(\mathrm{g})$ & MST $(\mathrm{g})$ & $\mathrm{R} /$ PA \\
\hline $\mathrm{T}_{1}($ TEST) & $18,04 \mathrm{c}$ & $3,96 \mathrm{~d}$ & $2,07 \mathrm{~b}$ & $0,51 \mathrm{~d}$ & $2,58 \mathrm{c}$ & $0,24 \mathrm{~b}$ \\
$\mathrm{~T}_{2}$ (Mi) & $16,88 \mathrm{c}$ & $4,09 \mathrm{~d}$ & $1,79 \mathrm{~b}$ & $0,49 \mathrm{~d}$ & $2,28 \mathrm{c}$ & $0,27 \mathrm{a}$ \\
$\mathrm{T}_{3}$ (calMi) & $16,44 \mathrm{c}$ & $3,93 \mathrm{~d}$ & $1,77 \mathrm{~b}$ & $0,51 \mathrm{~d}$ & $2,29 \mathrm{c}$ & $0,29 \mathrm{a}$ \\
$\mathrm{T}_{4}$ (cal) & $18,17 \mathrm{c}$ & $4,94 \mathrm{c}$ & $2,28 \mathrm{~b}$ & $0,61 \mathrm{~d}$ & $2,90 \mathrm{c}$ & $0,26 \mathrm{a}$ \\
$\mathrm{T}_{5}$ (COM) & $27,22 \mathrm{a}$ & $5,99 \mathrm{~b}$ & $8,93 \mathrm{a}$ & $1,50 \mathrm{~b}$ & $10,43 \mathrm{a}$ & $0,16 \mathrm{c}$ \\
$\mathrm{T}_{6}$ (COM-N) & $28,70 \mathrm{a}$ & $7,30 \mathrm{a}$ & $8,66 \mathrm{a}$ & $1,85 \mathrm{a}$ & $10,51 \mathrm{a}$ & $0,21 \mathrm{~b}$ \\
$\mathrm{~T}_{7}$ (COM-P) & $18,22 \mathrm{c}$ & $4,10 \mathrm{~d}$ & $2,01 \mathrm{~b}$ & $0,52 \mathrm{~d}$ & $2,53 \mathrm{c}$ & $0,25 \mathrm{a}$ \\
$\mathrm{T}_{8}$ (COM-K) & $29,85 \mathrm{a}$ & $6,26 \mathrm{~b}$ & $10,79 \mathrm{a}$ & $1,69 \mathrm{a}$ & $12,48 \mathrm{a}$ & $0,16 \mathrm{c}$ \\
$\mathrm{T}_{9}$ (COM-S) & $22,82 \mathrm{~b}$ & $5,37 \mathrm{c}$ & $7,44 \mathrm{a}$ & $1,23 \mathrm{~b}$ & $8,68 \mathrm{~b}$ & $0,16 \mathrm{c}$ \\
$\mathrm{T}_{10}$ (COM-cal) & $28,22 \mathrm{a}$ & $5,94 \mathrm{~b}$ & $8,23 \mathrm{a}$ & $1,22 \mathrm{~b}$ & $9,45 \mathrm{~b}$ & $0,14 \mathrm{c}$ \\
$\mathrm{T}_{11}$ (COM-cal-N) & $30,15 \mathrm{a}$ & $7,02 \mathrm{a}$ & $8,69 \mathrm{a}$ & $1,79 \mathrm{a}$ & $10,49 \mathrm{a}$ & $0,21 \mathrm{~b}$ \\
$\mathrm{~T}_{12}$ (COM-cal-P) & $18,66 \mathrm{c}$ & $4,39 \mathrm{~d}$ & $2,34 \mathrm{~b}$ & $0,55 \mathrm{~d}$ & $2,89 \mathrm{c}$ & $0,23 \mathrm{~b}$ \\
$\mathrm{~T}_{13}$ (COM-cal-K) & $25,40 \mathrm{~b}$ & $5,05 \mathrm{c}$ & $6,74 \mathrm{a}$ & $0,95 \mathrm{c}$ & $7,70 \mathrm{~b}$ & $0,14 \mathrm{c}$ \\
$\mathrm{T}_{14}$ (COM-cal-S) & $25,06 \mathrm{~b}$ & $6,00 \mathrm{~b}$ & $7,54 \mathrm{a}$ & $1,29 \mathrm{~b}$ & $8,83 \mathrm{~b}$ & $0,17 \mathrm{c}$ \\
\hline
\end{tabular}

Letras distintas na mesma coluna diferem entre si pelo teste Scott-Knott a 5\% de probabilidade. 
Tucci e Pinto (2003) trabalhando com adubação nitrogenada na produção de mudas de mogno, não observaram efeito das doses crescentes de $\mathrm{N}$ para as características matéria seca de caule e raiz, bem como para a altura da planta.

Vários outros trabalhos referentes à nutrição de espécies florestais mostraram que a omissão de $\mathrm{N}$ não afetou o crescimento das mesmas. É exemplo disso, os trabalhos de Duboc et al. (1996), com a espécie jatobá (Hymenaea coubaril); de Mendonça et al. (1999), com aroeira do sertáo (Myracrodruon urundeuva); e Venturin e Souza (2000) com a candiúva (Trema micrantha).

A omissão de $P$, na presença ou ausência de calagem $\left(T_{7}\right.$ e $\mathrm{T}_{12}$ respectivamente), limitou o crescimento em altura, diâmetro e a produçấo de matéria seca da parte aérea, raiz e total das mudas de mogno (Tabela 2). Tal fato é evidente quando se compara o tratamento completo $\left(\mathrm{T}_{5}\right)$ com os tratamentos com omissão de $\mathrm{P}\left(\mathrm{T}_{7}\right.$ e $\left.\mathrm{T}_{12}\right)$ e com a parcela testemunha, indicando ser o $\mathrm{P}$ o nutriente mais limitante ao crescimento da espécie.

Estes resultados corroboram com os encontrados por Siqueira e Franco (1988), que verificaram que a omissão de P limitou o crescimento em altura e diâmetro, e a produção de matéria seca da parte aérea, raiz e total, visto que a aplicação da adubação fosfatada trouxe resposta satisfatória ao crescimento da espécie Acacia mangium.

Barros (2001) relatou que a omissão do $\mathrm{P}$ afetou a produção de biomassa das mudas de mogno, pois os tratamentos com adubação fosfatada apresentaram as menores médias.

Resultados semelhantes à omissão de $\mathrm{P}$ como fator limitante, foram encontrados por Renó (1994) trabalhando com as espécies Caesalpinea ferrea, Senna multijuga, e Piptadenia gonoacantha; Silva e Muniz (1995) com Cedrella fissilis; Braga et al. (1995), com Acacia mangium, Tibouchina granulosa e Aspidosperma polyneurom e Mendonça et al. (1999) com Myracrodruon urundeuva.

Renó et al. (1993) concluíram que para o crescimento em altura do cedro (Cedrella fissilis), do jacaré (Piptadenia gonoacantha), do pau-ferro (Caesalpinea ferrea) e da canafístula (Senna multijuga) o fósforo foi limitante.

Carniel et al. (1993) observaram em resposta à adubação em campo que a embaúba (Cecropia sp.), o ipê-mirim (Stenolobium stans), o fedegoso (Senna macranthera), a cássia (Senna multijuga) e o angico amarelo (Peltophorum dubium) tiveram o crescimento afetado pela omissão de $\mathrm{P}$, com exceçẫo do ipê-mirim.

Dias et al. (1991) constataram que o fósforo é um nutriente limitante, para a produção de mudas de acácia (Acacia mangium), pois a adubação fosfatada favoreceu a produção da MSPA das mesmas. Duboc et al. (1996) verificaram que a produção de matéria seca da parte aérea das mudas de copaíba foi reduzida quando o teor de P no substrato era baixo. Este trabalho apresentou resultados semelhantes aos encontrados por Duboc et al. (1996), nos tratamentos com a omissão de fósforo ( $\mathrm{T}_{7} \mathrm{e} \mathrm{T}_{12}$ ) a produção de MSPA foi reduzida (Tabela 4).

Ao contrário do $\mathrm{P}$ (Tabela 2) que sua omissáo limitou a altura, o diâmetro, a MSPA, a MSR e a MST, a omissáo do $S$ $\left(\mathrm{T}_{9} \mathrm{e} \mathrm{T}_{14}\right)$ limitou apenas o crescimento em altura, diâmetro e a produção da matéria seca total das mudas de mogno. Porém, nem mesmo para essas características sua omissão foi táo limitante quanto à omissáo de $\mathrm{P}$, pois os valores destes tratamentos superaram aqueles onde $\mathrm{P}$ estava ausente $\left(\mathrm{T}_{7} \mathrm{e}\right.$ $\left.\mathrm{T}_{12}\right)$.

Os resultados encontrados neste experimento que mostram que a omissão do enxofre limitou apenas o crescimento em altura, diâmetro e a produção da matéria seca total das mudas de mogno, são semelhantes aos encontrados por Braga et al. (1995), que observaram que o crescimento em altura, diâmetro e produção da matéria seca total das plantas de quaresmeira (Tibouchina granulosa) foi comprometido com a omissão de $S$.

Na presença de calagem $\left(T_{8}\right)$ a omissão de potássio apresentou maior produção de matéria seca de raiz quando comparado ao tratamento completo $\left(\mathrm{T}_{5}\right)$, não influenciando as demais características de crescimento do mogno (Tabela 2). Porém, quando o $\mathrm{K}$ foi omitido sem a presença de calagem $\left(T_{13}\right)$, foi observado que houve uma diminuição no crescimento em altura e diâmetro, e na produção de matéria seca da parte aérea, raiz e total (Tabela 2).

Venturin et al. (1996) para a espécie Hymenaea coubaril (jatobá) e Duboc et al. (1996) para as espécies Hymenaea coubaril (jatobá) e Copaifera langsdorff (copaíba), também verificaram que a omissão de $\mathrm{K}$ na adubação não reduziu o crescimento em altura e diâmetro, e nem a produçáo da matéria seca da parte aérea das plantas. Para os referidos autores, a ausência de respostas destas espécies ao K parece estar relacionada com o baixo requerimento deste nutriente na fase de muda.

A calagem quando utilizada de forma isolada $\left(\mathrm{T}_{4}\right)$ ou combinada apenas com micronutrientes $\left(\mathrm{T}_{3}\right)$ reduziu, com exceção da relação raiz/parte aérea, as características de crescimento avaliadas neste estudo quando comparada ao tratamento completo $\left(\mathrm{T}_{5}\right)$ (Tabela 2 ).

Os resultados encontrados neste experimento que mostram a falta de resposta das mudas de mogno à calagem, diferem dos encontrados por Barros (2001) e Silva (2004), que observaram respostas positivas à aplicação da calagem para as características de crescimento das mudas de mogno. Vale ressaltar, entretanto, que a quantidade de $\mathrm{Ca}^{2+}+\mathrm{Mg}^{2+}$ presente no solo utilizado para compor o substrato, nos trabalhos dos referidos autores foi de 0,35 $\mathrm{cmol}_{\mathrm{c}} \mathrm{Kg}^{-1}$ e de 0,10 $\mathrm{cmol}_{\mathrm{c}} \mathrm{Kg}^{-1}$, respectivamente, e neste trabalho foi de $1,06 \mathrm{cmol}_{c} \mathrm{Kg}^{-1}$, a quantidade de cálcio e 
magnésio trocáveis diferiram entre eles, tal fato pode explicar a diferença encontrada entre os trabalhos.

Vários outros trabalhos com espécies florestais mostram que a calagem não influencia o desenvolvimento das plantas. $\mathrm{E}$ exemplo disso os trabalhos de Erasmo (1995); Gomes (1996) e Pacheco (1996).

Avaliando a formaçáo de mudas de Acacia holosericea e Acacia auriculiforms em resposta a calagem, P, K e S, Balieiro et al. (2001) não observaram resposta positiva em altura da planta, diâmetro do caule e peso da matéria seca da parte aérea para a adiçáo de corretivo, o que evidencia a tolerância das espécies à acidez do solo.

Pereira e Pereira (1987) observaram que a adição de calcário dolomítico tanto diretamente ao solo, como em sacos plásticos no viveiro, não teve efeito positivo no desenvolvimento de porta-enxertos de seringueira. Pelo contrário, esses autores observaram em experimento diretamente no solo, um efeito depressivo da calagem nas doses de 6 e 8 ton ha ${ }^{-1}$. Os autores acreditam ser esta a causa principal da reduçáo de seu desenvolvimento em altura.

Novais et al. (1980) concluíram que E. grandis e E saligna pouco responderam a calagem, entretanto, mostram um acentuado efeito da calagem sobre a altura da parte aérea. Os mesmos autores atribuíram este efeito a virtual ausência de cálcio e magnésio trocáveis na amostra de solo utilizado como substrato.

\section{CONTEÚDO DE NUTRIENTES NAS MUDAS}

A absorção de nutrientes pelas mudas de mogno foi afetada pela omissão de $\mathrm{P}$, pois o conteúdo dos macro $\mathrm{e}$ micronutrientes nos tratamentos com omissão deste nutriente $\left(\mathrm{T}_{7} \mathrm{e} \mathrm{T}_{12}\right)$ foi inferior ao conteúdo do tratamento completo (Tabelas 3 e 4).

A resposta do fósforo como fator limitante à absorção de macro e micronutrientes pelas mudas de mogno, foi observada no crescimento, onde as menores respostas em altura, diâmetro, e produção de matéria seca da parte aérea, raiz e total (Tabela 2), foram observadas nos tratamentos em que o fósforo foi omitido da adubação $\left(\mathrm{T}_{7} \mathrm{e} \mathrm{T}_{12}\right)$. Estes resultados demonstram a importância deste nutriente para a formação das mudas de mogno.

A importância do fósforo foi relatada também por Siqueira (1995), o qual verificou que embora as espécies florestais apresentem requerimentos nutricionais distintos, a ausência de nutrientes como o fósforo, geralmente náo permite o desenvolvimento das plantas, sendo exigidos em concentrações distintas em função da espécie. Para Lopes (1998) o fósforo é essencial para o crescimento das plantas e nenhum outro nutriente pode substituí-lo, sendo assim necessário para a planta completar seu ciclo normal de produção.
Segundo IPNI (2007), o fósforo é crucial no metabolismo das plantas, desempenhando papel importante na transferência de energia da célula, na divisão celular, no crescimento das células, na respiração e na fotossíntese.

Para Grant et al. (2001), as limitaçôes na disponibilidade de P no início do ciclo vegetativo podem resultar em restriçôes do desenvolvimento, das quais as plantas não se recuperam posteriormente, mesmo aumentando o suprimento de $\mathrm{P}$ a níveis adequados.

Respostas semelhantes às de fósforo, foram também observadas nos tratamentos que não foram adubados com todos os macronutrientes analisados neste estudo $\left(T_{1}, T_{2}\right.$, $\mathrm{T}_{3}$ e $\mathrm{T}_{4}$ ), uma vez que, o conteúdo dos nutrientes nestes tratamentos foi inferior ao tratamento completo (Tabelas 3 e 4).

A omissão do K na presença da calagem $\left(\mathrm{T}_{8}\right)$ afetou apenas o conteúdo do $\mathrm{K}$ e $\mathrm{Mn}$, em relaçáo ao tratamento completo. Entretanto, a omissão do $\mathrm{K}$ na ausência da calagem $\left(\mathrm{T}_{13}\right)$, afetou, com exceção do $\mathrm{Cu}$ e $\mathrm{Fe}$, o conteúdo de todos os nutrientes (Tabelas 3 e 4 ).

Os resultados encontrados neste trabalho que indicam a omissão do K (na presença ou ausência de calagem) como fator limitante à absorção deste pelas mudas de mogno, corrobora com os encontrados por Sarcinelli et al. (2004), que estudando os sintomas de deficiência nutricional em mudas de Acacia holosericea em resposta à omissão de macronutrientes, verificaram que as plantas que receberam tratamento com omissão de K apresentaram teores deste nutriente, nos filódios novos e velhos, cerca de dez vezes inferiores aos observados nas plantas que receberam soluçáo nutritiva completa, ou seja, apresentaram teores inferiores aos do tratamento completo.

A omissão de $\mathrm{K}$, na ausência da calagem, como fator limitante a absorçáo de $S$, foi observada também por Carniel et al. (1993) e Venturin et al. (1999) estudando o angico amarelo (Peltoforum dubium), e Duboc et al. (1996) a copaíba (Copaifera langsdorff).

A omissão do enxofre (na presença ou ausência da calagem), em relaçáo ao tratamento completo, reduziu o conteúdo de N, P, Ca, Mg, S, B, Mn e Zn (Tabelas 3 e 4). Estes resultados corroboram em partes com os encontrados por Duboc et al. (1996) e Venturin et al. (1999), os quais observaram que a omissão do enxofre reduziu, com exceção do $\mathrm{P}$, os teores dos nutrientes nas folhas das mudas de angicoamarelo e do jatobá (Hymenaea coubaril) com a omissáo de S. Marques et al. (2004) estudando as mesmas espécies, também observaram que as omissôes de $S$ da solução nutritiva proporcionaram aumento na concentraçáo de $\mathrm{P}$ nas folhas.

A resposta de $\mathrm{N}$ está relacionada à presença ou ausência da calagem, pois os resultados diferem em função dessa prática. Assim, na ausência de $\mathrm{N}$ na presença da calagem $\left(\mathrm{T}_{6}\right)$, os 
conteúdos de $\mathrm{N}, \mathrm{P}, \mathrm{Mg}$ e $\mathrm{S}$ foram reduzidos em relaçáo ao tratamento completo. No entanto, a omissão do $\mathrm{N}$ na ausência da calagem $\left(\mathrm{T}_{11}\right)$ reduziu apenas o conteúdo de $\mathrm{N}, \mathrm{Mg}$ e $\mathrm{S}$ (Tabelas 3 e 4).

Os resultados que indicam que a omissão de $\mathrm{N}$ reduz o conteúdo desse nutriente na parte aérea das mudas de mogno, em parte corroboram com os encontrados por Marques et al. (2004), que trabalhando com o paricá (Schizolobium amazonicum) concluíram que a omissão de $\mathrm{N}$ reduziu o teor desse nutriente no caule e nas raízes quando comparado ao tratamento completo, exceto nas folhas.

De posse desses resultados pode ser observado que os tratamentos com omissão de $\mathrm{N}\left(\mathrm{T}_{6}\right.$ e $\left.\mathrm{T}_{11}\right)$ apresentaram os maiores conteúdos de K (Tabela 3). Para Raij (1991), este fato pode estar associado ao fenômeno de competição que ocorre entre o nitrogênio e o potássio.

Tabela 3 - Conteúdo de macronutrientes na matéria seca da parte aérea em plantas de mogno (Swietenia macrophylla King.) aos 120 dias após transplantio.

\begin{tabular}{|c|c|c|c|c|c|c|}
\hline \multirow{2}{*}{ Tratamentos } & $\mathrm{N}$ & $\mathrm{P}$ & $\mathrm{K}$ & $\mathrm{Ca}$ & $\mathrm{Mg}$ & $S$ \\
\hline & \multicolumn{6}{|c|}{ g/unidade experimental } \\
\hline $\mathrm{T}_{1}$ (TEST) & $5,10 \mathrm{c}$ & $0,09 \mathrm{c}$ & $2,35 \mathrm{e}$ & $2,05 c$ & $0,21 d$ & $0,63 \mathrm{c}$ \\
\hline $\mathrm{T}_{2}(\mathrm{Mi})$ & $4,45 \mathrm{c}$ & $0,08 \mathrm{C}$ & $2,14 \mathrm{e}$ & $1,94 \mathrm{c}$ & $0,17 d$ & $0,46 \mathrm{c}$ \\
\hline $\mathrm{T}_{3}$ (calMi) & $4,47 \mathrm{c}$ & $0,07 \mathrm{c}$ & $1,85 \mathrm{e}$ & $1,69 \mathrm{c}$ & $0,22 \mathrm{~d}$ & $0,42 \mathrm{c}$ \\
\hline $\mathrm{T}_{4}$ (cal) & $5,16 \mathrm{c}$ & $0,09 \mathrm{c}$ & $2,52 \mathrm{e}$ & $2,35 \mathrm{c}$ & $0,23 d$ & $0,52 \mathrm{c}$ \\
\hline $\mathrm{T}_{5}(\mathrm{COM})$ & $24,61 \mathrm{a}$ & $1,29 a$ & $15,01 \mathrm{~b}$ & $9,21 \mathrm{a}$ & $1,18 b$ & $2,37 \mathrm{a}$ \\
\hline $\mathrm{T}_{6}(\mathrm{COM}-\mathrm{N})$ & $16,98 \mathrm{~b}$ & $0,87 \mathrm{~b}$ & $18,89 a$ & $9,41 \mathrm{a}$ & $0,97 \mathrm{c}$ & $1,83 \mathrm{~b}$ \\
\hline $\mathrm{T}_{7}(\mathrm{COM}-\mathrm{P})$ & $5,38 \mathrm{c}$ & $0,10 \mathrm{C}$ & $2,48 \mathrm{e}$ & $1,64 \mathrm{c}$ & $0,22 \mathrm{~d}$ & $0,48 \mathrm{c}$ \\
\hline $\mathrm{T}_{8}(\mathrm{COM}-\mathrm{K})$ & $28,72 \mathrm{a}$ & $1,18 \mathrm{a}$ & $9,69 \mathrm{c}$ & $11,35 a$ & $1,51 \mathrm{a}$ & $2,33 a$ \\
\hline $\mathrm{T}_{9}(\mathrm{COM}-\mathrm{S})$ & $16,68 b$ & $0,76 \mathrm{~b}$ & $14,64 \mathrm{~b}$ & $8,33 \mathrm{~b}$ & $0,98 \mathrm{c}$ & $1,62 b$ \\
\hline $\mathrm{T}_{10}$ (COM-cal) & $18,81 b$ & $1,15 \mathrm{a}$ & $14,53 \mathrm{~b}$ & $8,32 \mathrm{~b}$ & $0,89 \mathrm{c}$ & $1,91 b$ \\
\hline $\mathrm{T}_{11}(\mathrm{COM}-\mathrm{cal}-\mathrm{N})$ & $17,21 \mathrm{~b}$ & $1,17 \mathrm{a}$ & $15,05 b$ & $9,60 \mathrm{a}$ & $0,90 \mathrm{c}$ & $1,74 b$ \\
\hline $\mathrm{T}_{12}(\mathrm{COM}-\mathrm{cal}-\mathrm{P})$ & $5,38 \mathrm{c}$ & $0,12 \mathrm{c}$ & $2,82 \mathrm{e}$ & $1,89 \mathrm{c}$ & $0,19 d$ & $0,45 \mathrm{c}$ \\
\hline $\mathrm{T}_{13}(\mathrm{COM}-\mathrm{cal}-\mathrm{K})$ & 16,92 b & $0,71 \mathrm{~b}$ & $6,65 d$ & $6,37 \mathrm{~b}$ & $0,80 \mathrm{c}$ & $1,64 b$ \\
\hline $\mathrm{T}_{14}(\mathrm{COM}-\mathrm{cal}-\mathrm{S})$ & $17,45 \mathrm{~b}$ & $0,77 \mathrm{~b}$ & $14,60 \mathrm{~b}$ & $7,49 \mathrm{~b}$ & $0,75 \mathrm{c}$ & $1,65 b$ \\
\hline
\end{tabular}

Letras distintas na mesma coluna diferem entre si pelo teste Scott-Knott a 5\% de probabilidade.

Tabela 4 - Conteúdo de micronutrientes na matéria seca da parte aérea em plantas de mogno (Swietenia macrophylla King.) aos 120 dias após transplantio.

\begin{tabular}{|c|c|c|c|c|c|}
\hline \multirow{2}{*}{ Tratamentos } & B & $\mathrm{Cu}$ & $\mathrm{Fe}$ & $\mathrm{Mn}$ & $\mathrm{Zn}$ \\
\hline & \multicolumn{5}{|c|}{ g/unidade experimental } \\
\hline $\mathrm{T}_{1}$ (TEST) & $0,01 \mathrm{c}$ & $0,0006 \mathrm{~b}$ & $2,36 \mathrm{a}$ & $0,04 \mathrm{c}$ & $0,03 \mathrm{c}$ \\
\hline $\mathrm{T}_{2}(\mathrm{Mi})$ & $0,02 \mathrm{c}$ & $0,0007 \mathrm{~b}$ & $0,20 \mathrm{a}$ & $0,02 \mathrm{c}$ & $0,02 \mathrm{c}$ \\
\hline $\mathrm{T}_{3}$ (calMi) & $0,01 \mathrm{c}$ & $0,0004 \mathrm{~b}$ & $0,11 \mathrm{a}$ & $0,01 \mathrm{c}$ & $0,01 \mathrm{c}$ \\
\hline $\mathrm{T}_{4}$ (cal) & $0,01 \mathrm{c}$ & $0,0003 b$ & $0,10 \mathrm{a}$ & $0,02 \mathrm{c}$ & $0,01 \mathrm{c}$ \\
\hline $\mathrm{T}_{5}(\mathrm{COM})$ & $0,05 \mathrm{a}$ & $0,0007 \mathrm{~b}$ & $0,38 \mathrm{a}$ & $0,14 \mathrm{a}$ & $0,04 \mathrm{a}$ \\
\hline $\mathrm{T}_{6}(\mathrm{COM}-\mathrm{N})$ & $0,05 \mathrm{a}$ & $0,0008 \mathrm{~b}$ & $0,26 a$ & $0,12 \mathrm{a}$ & $0,04 \mathrm{a}$ \\
\hline $\mathrm{T}_{7}(\mathrm{COM}-\mathrm{P})$ & $0,02 \mathrm{c}$ & $0,0003 b$ & $0,08 \mathrm{a}$ & $0,02 \mathrm{c}$ & $0,01 \mathrm{c}$ \\
\hline $\mathrm{T}_{8}(\mathrm{COM}-\mathrm{K})$ & $0,06 \mathrm{a}$ & $0,0007 \mathrm{~b}$ & $0,37 a$ & $0,10 \mathrm{~b}$ & $0,05 \mathrm{a}$ \\
\hline $\mathrm{T}_{9}(\mathrm{COM}-\mathrm{S})$ & $0,03 \mathrm{~b}$ & $0,0019 a$ & $0,19 a$ & $0,08 \mathrm{~b}$ & $0,04 \mathrm{~b}$ \\
\hline $\mathrm{T}_{10}$ (COM-cal) & $0,05 \mathrm{a}$ & $0,0019 a$ & $0,22 \mathrm{a}$ & $0,13 \mathrm{a}$ & $0,04 b$ \\
\hline $\mathrm{T}_{11}(\mathrm{COM}-\mathrm{cal}-\mathrm{N})$ & $0,06 \mathrm{a}$ & $0,0020 \mathrm{a}$ & $0,28 \mathrm{a}$ & $0,13 a$ & $0,05 \mathrm{a}$ \\
\hline $\mathrm{T}_{12}$ (COM-cal-P) & $0,02 \mathrm{c}$ & $0,0006 \mathrm{~b}$ & $0,08 \mathrm{a}$ & $0,02 \mathrm{c}$ & $0,01 \mathrm{c}$ \\
\hline $\mathrm{T}_{13}(\mathrm{COM}-\mathrm{cal}-\mathrm{K})$ & $0,03 \mathrm{~b}$ & $0,0009 \mathrm{~b}$ & $0,16 \mathrm{a}$ & $0,07 \mathrm{~b}$ & $0,03 \mathrm{~b}$ \\
\hline $\mathrm{T}_{14}$ (COM-cal-S) & $0,04 \mathrm{~b}$ & $0,0008 b$ & $0,17 \mathrm{a}$ & $0,09 \mathrm{~b}$ & $0,04 \mathrm{~b}$ \\
\hline
\end{tabular}

Letras distintas na mesma coluna diferem entre si pelo teste Scott-Knott a 5\% de probabilidade. 
A competição entre o $\mathrm{N}$ e o K foi observada por Santos (2006), que verificou que as mudas de mogno não apresentaram diferença significativa na absorção de macronutrientes em função das doses crescentes de $\mathrm{N}$, com exceção da absorção de K que foi favorecida pelas menores doses de N, observando redução na absorção de K para as maiores doses de $\mathrm{N}$ aplicadas no substrato. Silva e Muniz (1995) verificaram também a interação entre esses nutrientes, uma vez que, trabalhando com mudas de peroba-rosa (Aspidosperma polyneuron) constataram que a omissão de nitrogênio proporcionou maior disponibilidade de potássio nas raízes.

Resultados semelhantes à interação entre $\mathrm{N}$ e $\mathrm{K}$, foram observados por Tucci et al. (2002), que observaram respostas positivas em altura e em matéria seca da parte aérea à adubação nitrogenada na ausência potássica. Entretanto, na presença da potássica, não observaram mais resposta ao $\mathrm{N}$. Para os autores a interação negativa tem sido atribuída ao efeito inibitório do nitrogênio pela aplicação de potássio.

\section{CONCLUSÕES}

Os resultados obtidos nas condiçóes experimentais desta pesquisa para a formação das mudas de mogno permitem concluir que:

É necessária a correção conjunta da acidez e da fertilidade do solo para solos ácidos e de baixa fertilidade natural, mesmo que o teor de matéria orgânica seja considerado alto;

Dos nutrientes, P é o que mais limita o crescimento de plantas de mogno, bem como a absorção dos outros nutrientes;

Não há necessidade de adiçấo de K para o crescimento das plantas quando se realiza calagem;

$\mathrm{Na}$ ausência de calagem a adição de K deve ser recomendada; $\mathrm{e}$

$\mathrm{O}$ requerimento nutricional do mogno em macronutrientes obedece à ordem decrescente de: $\mathrm{P}>\mathrm{S}>\mathrm{K}>\mathrm{N}$.

\section{BIBLIOGRAFIA CITADA}

Balieiro, F. de C.; Oliveira, I.G. de; Dias, L.E. 2001. Formation of seedlings of Acacia holosericea and Acacia auriculiforms: response to liming, phosphorus, potassium and sulfur. Rev. Árvore 25(2): 183-191 (in Portuguese, with abstract in English).

Barros, J.G. 2001. Liming and fertilization to the formation of seedlings of mogno (Swietenia macrophylla King). Dissertação de Mestrado, UFAM, Manaus, Amazonas. 64pp (in Portuguese).

Braga, F.de A; Vale, F.R; Ventorim, N; Aubert, E.; Lopes, G.de A. 1995. Nutritional requirements of four forestry species. Árvore 19 (1): 18-31 (in Portuguese, with abstract in English).

Carniel, T.; Lima, H.N.; Vale, F.R. do; Siqueira, J.O.; Curi, N.; Gomes, R.J. 1993. Response to fertilization in the field of five native species of southeastern Brazil. IN: CONGRESSO BRASILEIRO DE CIÊNCIA DO SOLO 24, Goiânia: SBCS. p209-210 (in Portuguese).
CFSEMG. 1999. Recommendations for the use of fertilizers and in Minas Gerais. 5a. Aprox. Viçosa, Minas Gerais. 359pp (in Portuguese).

Dias, L.E.; Alvarez, V.H.V.; Brienza, J.S. 1991. Formation of seedlings of Acacia mangium Willd: II: Responses to nitrogen and potassium. Rev. Arvore 15: (1) 12-22 (in Portuguese, with abstract in English).

Duboc, E.; Venturin, N.; Vale, F.R do; Davide A .C. 1996. Nutrition jatoba (Hymenaea coubaril L. var. Stilbocarpa (Hayne) Lee et Lang). Cerne, 2(1): 138-152 (in Portuguese, with abstract in English).

EMBRAPA. 1999. Handbook of chemical analysis of soils, plants and fertilizers. Brasília. 370pp (in Portuguese).

Erasmo, E.A.L. 1995. Growth, mineral nutrition and response to liming in Senna obstusifolia (L.). Irvin \& Barneby. Dissertação Mestrado em Agronomia, UNESP, Jaboticabal, São Paulo. 88pp (in Portuguese).

Gomes, F.P. 1996. Nutritional requirement of peach palm (Bactris gasipes $H B K)$ in representative soils of southern Bahia. Dissertação - Mestrado, UFBa, Salvador, Bahia. 49pp (in Portuguese).

Grant, C.A.; Flaten, D.N.; Tomasiewicz, D.J.; Sheppard, S.C.. 2001. The importance of phosphorus in the initial development of plant. POTAFOS- Associação Brasileira para Pesquisa da Potassa e do Fosfato. Informaçóes Agronômicas, n.95, 5pp (in Portuguese)

IPNI. 2007. International Plant Nutrition Institute. Importancia del fósforo em el suelo. (/www.ppi-far.org). Acesso: 22/07/2006.

Lopes, A.S. 1998. Handbook of soil identification. Tradução e adaptação de Alfredo Scheid Lopes. São Paulo, ANDA/ POTAFOS. 153pp. (in Portuguese).

Malavolta, E.; Vitti, G.C.; Oliveira, S.A. 1997. Assessment of nutritional status of plants: principles and applications. $2^{\mathrm{a}}$. ed. Piracicaba: POTAFOS. 319pp (in Portuguese).

Marques, T.C.; Carvalho, J.G.; Lacerda, M.P.C; Mota, P.E.F. 2004. Nutritional requirements of paricá (Shizolobium amazonicum, Herb.) seedling phase. Cerne, 10(2): 167-183 (in Portuguese, with abstract in English).

Mendonça, A.V.R.; Nogueira, F.D.; Venturim, N.; Souza, J.S. 1999. Nutritional requirements of Myracrodruon urundeuval Fr. All (Aroeira do sertáo). Cerne, 5(2): 67-75 (in Portuguese, with abstract in English).

Novais, R.F; Gomes, J.M.; Borges, E.E.L.; Rocha, D. 1980. Liming and mineral in the production of seedlings eucalyptus (Eucalyptus grandis W. Hill ex Maiden). Arvore 6 (1): 45-51 (in Portuguese, with abstract in English).

Oliveira, A.J.; Silva, A.; Schwengber, D.; Duarte, O. 1997. Response of seedlings of Dinizia excelsa Ducke to nitrogen and phosphorus. Pesq. Agropec. Bras. 33(9): 1503-1507 (in Portuguese, with abstract in English).

Pacheco, R.G. 1996. Growth of seedlings of peach palm (Bactris gasipes $H B K)$ in response to liming and relations calcium / magnesium in the soil and answers relations / nitrate and ammonium in nutrient solutions. Dissertação de Mestrado, UFV, Viçosa, Minas Gerais. 108pp (in Portuguese). 
Pereira, A.V.; Pereira, E.B.C. 1987. Responses from rootstocks to lime tree. Rev. Bras. de Ci. do Solo 11:333-336 (in Portuguese, with abstract in English).

Raij, B.V. 1991. Soil fertility and fertilization. Piracicaba: Ed. Agronômica Ceres Ltda. 343pp. (in Portuguese).

Renó, N.B. 1994. Nutritional requirements and response to phosphorus and mycorrhizal fungi of native tree species in southeastern Brazil. Dissertação de Mestrado, UFAL, Lavras, Minas Gerais. 64pp. (in Portuguese).

Renó, N.B.; Vale F.R.; Curi, N.; Siqueira, J.O. 1993. Nutritional requirements of four native species. In: Brazilian Congress of Soil Science, 24. Goiânia, Resumos..., SBCS. p211-212 (in Portuguese).

Sá, J.C.M. 1993. Management of soil fertility in no-tillage. In: EMBRAPA. Centro Nacional de Pesquisas de Trigo (Passo Fundo - RS). Plantio direto no Brasil. p37-60 (in Portuguese).

Sá, J.C.M. 2001. Evolution of organic matter in soil tillage. In: IV Curso sobre aspectos básicos de fertilidade de solo em plantio direto: 4, 2001. Resumo de palestras... Passo Fundo: Aldeia Norte, p5-20 (in Portuguese).

Santos, R.A.C. 2006. Effects of increasing doses of nitrogen, phosphorus and potassium in seedlings of mahogany (Sweitenia macrophylla) and stick (Ochroma pyramidale) and selection of extractants of phosphorus and potassium. Dissertação de Mestrado, UFAM , Manaus, Amazonas. 64pp (in Portuguese).

Santos, H.P.; Tom, G.O. 2003. Availability of nutrients and organic matter on the basis of cropping systems and soil management. Rev. Ciência Rural 33(3): 477-486 (in Portuguese, with abstract in English).

Santos, H.P.; Tom, G.O.; Lhamby, J.C.B. 1995. Tillage versus conventional: effect on soil fertility and yield of crops in crop rotation with barley. Rev. Bras. de Ci. do Solo, 19 (3): 449- 454 (in Portuguese, with abstract in English).

Sarcinelli, T.S.; Ribeiro Júnior, E.S.; Dias, L.E.; Lynch, L.S. 2004. Nutrient deficiency symptoms in seedlings of Acacia holosericea in response to the omission of macronutrients. Arvore 28 (2): 173-181 (in Portuguese, with abstract in English).

Sena, J. dos S. 2008. Effect of liming and correction of Ca and $\mathrm{Mg}$ in the soil on the growth of seedlings of Dinizia excelsa Ducke, Cedrela odorata L. and Swietenia macrophylla King.). Dissertação de Mestrado, UFAM, Manaus, Amazonas. 87pp (in Portuguese).

Silva, A.R.M. 2004. Liming for the production of seedlings of mogno (Swietenia macrophylla King.) and cedro (Ceiba pentandra L. Gaertn). Dissertação de Mestrado, UFAM, Manaus, Amazonas. 53pp (in Portuguese).

Silva, A.R.M.; Tucci, C.A.F.; Lima, H. N.; Figueiredo, A. F. 2007. Growing doses of liming on mogno (Swietenia macrophylla King) in seedling formation. Acta Amazonica, 37(2): 195-200 (in Portuguese, with abstract in English).
Silva, M.A.G.; Muniz, A.S. 1995. Nutritional requirements of cedar (Cedrella fissilis Velloso) in nutrient solution. Arvore 19 (3): 415425 (in Portuguese, with abstract in English).

Silva Júnior, C.H. 2006. Response to fertilization with nitrogen, phosphorus and potassium in the formation of seedlings of mahogany (Swietenia macrophylla King.) and kapok (Ceiba pentrandra). Dissertação de Mestrado, UFAM, Manaus, Amazonas. 56pp. (in Portuguese).

Siqueira, J.O.; Franco, A.A. 1988. Biotechnology of soil: fundamentals and prospects. Brasília: Ministério da Educação. 235pp (in Portuguese).

Siqueira, J.O. 1995. Aspects of soils, plant nutrition and microbiology in the implementation of riparian. Belo Horizonte, Minas Gerais: CEMIG. 23pp (in Portuguese).

Tucci, C.A.F.; Pinto, F. 2003. Nitrogen in the production of seedlings of mahogany. In: Brazilian Congress of Soil Science, 29. Ribeirão Preto. CD-ROM do 29 CBCS (in Portuguese).

Tucci, C.A.F.; Hara, F.A.S; Freitas, R. O. de. 2002. Fertilizing and liming for the formation of seedlings of kapok (Ceiba pentandra (L.) Gaertn). Rev. da Universidade do Amazonas: Série C. Agrárias 10(1/2):29-31 (in Portuguese, with abstract in English).

Tucci, C. A. F. 1991. Phosphorus availability in soils of the Amazon. Tese (Doutorado em Solos, UFV, Viçosa, Minas Gerais. 142pp (in Portuguese).

Venturin, N.; Duboc, E.; Vale, F.R.; Davide, A.C. 1996. Fertilization of seedlings of Copaifera langsdorffi (Oil Copaiba). Cerne 2(2): 31-47 (in Portuguese, with abstract in English).

Venturim, N.; Duboc, E.; Vale, F.R.do; Davide, A.C. 1999. Adubação mineral do angico-amarelo (Peltophorum dubium (Spreng.) Taub.). Pesq. Agropec. Bras. 34(3): 441-448 (in Portuguese, with abstract in English).

Venturim N.; Souza, P. A. 2000. Nutritional evaluation of candiúva (Trema micrantha Blume L.) in greenhouse. Rev. Floresta 29(1/2): 15-26 (in Portuguese, with abstract in English).

Wietholter, S. 2000. Management of soil fertility in no-tillage system: experience in the states of Rio Grande do Sul and Santa Catarina. In: Brazilian meeting of soil fertility and plant nutrition, 25; Brazilian meeting on mycorrhizae, 8; Brazilian symposium on soil microbiology, 6; Reunion brasileira de Biologia soil, 3. Santa Maria: Centro de Ciências Rurais do Departamento de Solos, UFSM. CD-ROM. (in Portuguese).

Recebido em 23/05/2008

Aceito em 10/11/2009 